\title{
Pohozaev-type inequalities and their applications for elliptic equations
}

\author{
Bingyu Kou* and Tianqing An
}

"Correspondence: koubei@163.com College of Science, Hohai University, Nanjing, 210098, China

\begin{abstract}
In this paper we derive the Pohozaev-type inequalities for $p$-Laplacian equations and weighted quasi-linear equations and then prove some non-existence results for the positive solutions of these equations in a class of domains that are more general than star-shaped ones.
\end{abstract}

Keywords: Pohozaev-type inequality; p-Laplacian; quasi-linear equation; positive solutions; non-star-shaped domain; non-existence

\section{Introduction}

This paper is mainly concerned with the elliptic equation

$$
\begin{cases}-\Delta_{p} u=f(x, u), & \text { in } \Omega, \\ u=0, & \text { on } \partial \Omega,\end{cases}
$$

where $\Delta_{p}=\operatorname{div}\left(|\nabla u|^{p-2} \nabla u\right), f(x, u): R^{N} \times R^{1} \rightarrow R^{1}$ is continuous, and $\Omega \subset \mathbb{R}^{n}(n \geq 3)$ is a bounded domain with smooth boundary. We will establish the Pohozaev-type inequality for the solutions of (1.1) and then discuss the non-existence of positive solutions of the problem in the non-star-shaped domains. We also discuss a similar topic for the following weighted quasi-linear elliptic equation:

$$
\begin{cases}-\operatorname{div}\left(|x|^{-a p}|\nabla u|^{p-2} \nabla u\right)=f(x, u) & \text { in } \Omega \\ u=0 & \text { on } \partial \Omega .\end{cases}
$$

Recall that in the famous paper [1], Pohozaev considered the following elliptic boundary value problem:

$$
\begin{cases}-\Delta u=f(u) & \text { in } \Omega, \\ u=0 & \text { on } \partial \Omega\end{cases}
$$

where $f \in C\left(R^{1}, R^{1}\right), \Omega \subset \mathbb{R}^{n}(n \geq 3)$ is a domain with smooth boundary. Let $F(x)=$ $\int_{0}^{x} f(s) d s$, and let $v(x)$ be the unit outward normal to $\partial \Omega$ at $x$. He proved the following famous identity.

(c) The Author(s) 2017. This article is distributed under the terms of the Creative Commons Attribution 4.0 International License (http://creativecommons.org/licenses/by/4.0/), which permits unrestricted use, distribution, and reproduction in any medium, provided you give appropriate credit to the original author(s) and the source, provide a link to the Creative Commons license, and indicate if changes were made. 
Theorem A (Pohozaev identity, [1]) Suppose that $u \in C^{2}(\Omega) \cap C^{1}(\bar{\Omega})$ is a solution of (1.3). Then

$$
\begin{aligned}
(2 & -n) \int_{\Omega} u f(u) d x+2 n \int_{\Omega} F(u) d x \\
= & \int_{\partial \Omega}\langle x, v(x)\rangle\left|\frac{\partial u}{\partial v}\right|^{2} d s .
\end{aligned}
$$

Based on this identity, Pohozaev obtained a remarkable non-existence result for the following elliptic boundary value problem under the conditions that $\Omega$ is star-shaped and $\alpha \geq \frac{n+2}{n-2}, \lambda \leq 0$.

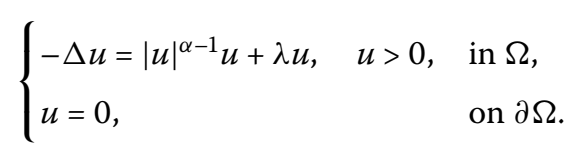

Since then, many new results on this topic have appeared. Some of them generalized Pohozaev's results to the more general equations, such as quasi-linear elliptic, polyharmonic equations, fractional differential equations. Others considered the case of domains more general than star-shaped ones. See [2-9] and the references therein. In [10], Bahri and Coron proved that if $\Omega$ is a smooth domain with non-trivial topology, equation (1.5) may have solutions. Dancer [11] and Ding [12] constructed the examples of contractible domains on which (1.5) has a solution. Therefore, it is interesting to discuss the problem on non-star-shaped contractible domains.

In 1989, Guedda and Veron [13] established the Pohozaev identity of the solutions of (1.1) and got the non-existence results. In [14], Isaia generalized the Pohozaev identity to a non-existence result of higher-order regular strong solutions of (1.1). In [15], Takáč and Il'yasov improved the well-known regularity results of the weak solutions of $\mathrm{p}$-Laplacian equation from $[16,17]$ and, using the new regularity results for the Dirichlet and Neumann problem, established and proved the Pohozaev-type identity. In [18], Bartsch, Peng and Zhang generalized the non-existence result to the more general problem (1.2). It is also interesting to discuss some special cases of (1.1), such as $f(x, u)=\lambda u^{q-1}+u^{s-1}, f(x, u)=$ $p(x) u^{\alpha}+q(x) u^{\beta}$, etc. See, for example, [19-22].

In this paper we also discuss the non-existence of the positive solution of (1.1) and (1.2). However, our method is different from all of the above work. Instead of the Pohozaev identities, we establish a kind of inequalities, named Pohozaev-type inequalities, which have the same effects as Pohozaev identities, and then prove some non-existence results for the positive solution of (1.1) and (1.2) on non-star-shaped domains.

\section{The p-Laplacian equations}

In this section, we consider the p-Laplacian equations (1.1). Firstly we give a lemma.

Lemma 2.1 Assume that $V(x)=\left(V_{1}(x), \ldots, V_{n}(x)\right)$ is a $C^{1}$ vector field on $\mathbb{R}^{n}$ and $u \in$ $W_{0}^{1, p}(\Omega) \cap C^{1}(\bar{\Omega})$ is a solution of (1.1). Then

$$
\int_{\Omega} u \operatorname{div}\left(|\nabla u|^{p-2} V(x)\right) d x=-\int_{\Omega}|\nabla u|^{p-2}\langle V(x), \nabla u| d x
$$


and

$$
\int_{\Omega} F(x, u) \operatorname{div} V(x) d x+\int_{\Omega} F_{1}(x, u) d x=-\int_{\Omega} f(u)\langle V(x), \nabla u\rangle d x,
$$

where $F(x, t)=\int_{0}^{t} f(x, s) d s, F_{1}(x, t)=\sum_{i=1}^{n} V_{i} \frac{\partial F(x, t)}{\partial x_{i}}$.

Proof By the divergence theorem and the fact $u(x)=0$ and $F(x, u)=0$ for $x \in \partial \Omega$, we have the following results:

$$
\begin{aligned}
0 & =\int_{\partial \Omega}\left\langle u(x)|\nabla u|^{p-2} V(x), v(x)\right\rangle d s=\int_{\Omega} \operatorname{div}\left(u(x)|\nabla u|^{p-2} V(x)\right) d x \\
& =\int_{\Omega} u \operatorname{div}\left(|\nabla u|^{p-2} V(x)\right) d x+\int_{\Omega}\left\langle|\nabla u|^{p-2} V(x), \nabla u\right\rangle d x \\
& =\int_{\Omega} u \operatorname{div}\left(|\nabla u|^{p-2} V(x)\right) d x+\int_{\Omega}|\nabla u|^{p-2}\langle V(x), \nabla u\rangle d x,
\end{aligned}
$$

and because $V_{i} f(x, u) \frac{\partial u}{\partial x_{i}}=V_{i}\left(\frac{\partial F}{\partial x_{i}}-\int_{0}^{u} \frac{\partial f(x, s)}{\partial x_{i}} d s\right)$, we have

$$
\begin{aligned}
& -\sum_{i=1}^{n} \int_{\Omega} V_{i} f \frac{\partial u}{\partial x_{i}} d x \\
& =-\sum_{i=1}^{n}\left[\int_{\Omega}\left(\frac{\partial}{\partial x_{i}}\left(V_{i} F\right)-\frac{\partial V_{i}}{\partial x_{i}} F\right) d x-\int_{\Omega} V_{i} \frac{\partial F}{\partial x_{i}} d x\right] \\
& =-\int_{\partial \Omega} F(x, u)\langle V(x), v(x)\rangle d s+\int_{\Omega} F(x, u) \operatorname{div}(V(x)) d x+\int_{\Omega} F_{1}(x, u) x \\
& =\int_{\Omega} F(x, u) \operatorname{div}(V(x)) d x+\int_{\Omega} F_{1}(x, u) x .
\end{aligned}
$$

The proof is complete.

Based on Lemma 2.1, we can derive a Pohozaev-type inequality for the solutions of (1.1).

Theorem 2.2 (Pohozaev-type inequality) Let $V(x)$ be a linear vector field on $\mathbb{R}^{n}$ with the form

$$
V(x)=\left(\begin{array}{ccc}
a_{11} & \cdots & a_{1 n} \\
\vdots & \vdots & \vdots \\
a_{n 1} & \cdots & a_{n n}
\end{array}\right) x
$$

Suppose that $V(x)$ satisfies $\operatorname{div} V(x)=n$ and $\langle V(x), x\rangle>0$ for $\forall x \in \mathbb{R}^{n} \backslash\{0\}$. If $u \in W_{0}^{1, p}(\Omega) \cap$ $C^{1}(\bar{\Omega})$ is a solution of $(1.1)$, then

$$
\begin{aligned}
& (p \mu-n) \int_{\Omega} u f(x, u) d x+p n \int_{\Omega} F(x, u) d x+p \int_{\Omega} F_{1}(x, u) d x \\
& \geq(p-1) \int_{\partial \Omega}\langle V(x), v(x)\rangle\left|\frac{\partial u}{\partial v}\right|^{p} d s,
\end{aligned}
$$

where $\mu=\sup _{|x| \neq 0} \frac{\langle V(x), x\rangle}{|x|^{2}}$. 
Proof It is easy to see that

$$
0<\langle V(x), x\rangle<\mu|x|^{2}, \quad \forall x \in \mathbb{R}^{n} \backslash\{0\}
$$

We multiply the equation $-\Delta_{p} u=f(x, u)$ by $\langle V(x), \nabla u\rangle$, and then we integrate in $\Omega$. By the divergence theorem and Lemma 2.1, the right-hand side is

$$
\begin{aligned}
& -\int_{\Omega} f(x, u)\langle V(x), \nabla u\rangle d x \\
& =-\int_{\Omega} \sum_{i=1}^{n} f(x, u) V_{i}(x) \frac{\partial u}{\partial x_{i}} \\
& =\int_{\Omega} F(x, u) \operatorname{div} V(x) d x+\int_{\Omega} F_{1}(x, u) d x .
\end{aligned}
$$

The left-hand side is

$$
\begin{aligned}
& \int_{\Omega} \operatorname{div}\left(|\nabla u|^{p-2} \nabla u\right)\langle V(x), \nabla u\rangle d x \\
& =\sum_{j=1}^{n} \int_{\Omega} \frac{\partial}{\partial x_{j}}\left(|\nabla u|^{p-2} \frac{\partial u}{\partial x_{j}}\right)\left(\sum_{i=1}^{n} V_{i}(x) \frac{\partial u}{\partial x_{i}}\right) d x \\
& =\sum_{j=1}^{n} \int_{\Omega} \frac{\partial}{\partial x_{j}}\left(|\nabla u|^{p-2} \frac{\partial u}{\partial x_{j}} \sum_{i=1}^{n} V_{i}(x) \frac{\partial u}{\partial x_{i}}\right)-|\nabla u|^{p-2} \frac{\partial u}{\partial x_{j}} \frac{\partial}{\partial x_{j}}\left(\sum_{i=1}^{n} V_{i}(x) \frac{\partial u}{\partial x_{i}}\right) d x .
\end{aligned}
$$

Following this, we have

$$
\begin{aligned}
= & \int_{\partial \Omega}\left\langle V(x),\left.v(x)|| \nabla u\right|^{p} d s-\sum_{j=1}^{n} \int_{\Omega}|\nabla u|^{p-2} \frac{\partial u}{\partial x_{j}}\left(\sum_{i=1}^{n} a_{i j} \frac{\partial u}{\partial x_{i}}+\sum_{i=1}^{n} V_{i}(x) \frac{\partial^{2} u}{\partial x_{i} \partial x_{j}}\right)\right. \\
= & \int_{\partial \Omega}\langle V(x), v(x)\rangle|\nabla u|^{p} d s+\int_{\Omega} u \operatorname{div}\left(|\nabla u|^{p-2} V(\nabla u)\right) \\
& -\sum_{j=1}^{n} \int_{\Omega}|\nabla u|^{p-2} \frac{\partial u}{\partial x_{j}}\left(\sum_{i=1}^{n} V_{i} \frac{\partial^{2} u}{\partial x_{i} \partial x_{j}}\right) \\
= & \int_{\partial \Omega}\left\langle V(x),\left.v(x)|| \nabla u\right|^{p} d s-\int_{\Omega}|\nabla u|^{p-2}\langle V(\nabla u), \nabla u\rangle d x-\frac{1}{p} \sum_{i=1}^{n} \int_{\Omega} V_{i}(x) \frac{\partial}{\partial x_{i}}\left(|\nabla u|^{p}\right) d x\right. \\
= & \int_{\partial \Omega}\langle V(x), v(x)\rangle|\nabla u|^{p} d s-\int_{\Omega}|\nabla u|^{p-2}\langle V(\nabla u), \nabla u\rangle d x \\
& -\frac{1}{p} \int_{\partial \Omega}\langle V(x), v(x)\rangle|\nabla u|^{p} d s+\frac{1}{p} \sum_{i=1}^{n} a_{i i} \int_{\Omega}|\nabla u|^{p} d x .
\end{aligned}
$$

Comparing the left- and right-hand sides, we get the following identity:

$$
\begin{aligned}
& \left(1-\frac{1}{p}\right) \int_{\partial \Omega}|\nabla u|^{p}\langle V(x), v(x)\rangle d s \\
& =-\frac{1}{p} \sum_{i=1}^{n} a_{i i} \int_{\Omega}|\nabla u|^{p} d x+\int_{\Omega}|\nabla u|^{p-2}\langle V(\nabla u), \nabla u\rangle d x+\int_{\Omega} F \operatorname{div} V(x) d x
\end{aligned}
$$




$$
\begin{aligned}
= & -\frac{1}{p} \sum_{i=1}^{n} a_{i i} \int_{\Omega} u f(x, u) d x+\int_{\Omega}|\nabla u|^{p-2}\langle V(\nabla u), \nabla u| d x+\sum_{i=1}^{n} a_{i i} \int_{\Omega} F(x, u) d x \\
& +\int_{\Omega} F_{1}(x, u) d x .
\end{aligned}
$$

Because $0<\langle V(x), x\rangle \leq \mu|x|^{2}$, we know that

$$
0<\langle V(\nabla u), \nabla u\rangle \leq \mu|\nabla u|^{2}
$$

Thus,

$$
\int_{\Omega}|\nabla u|^{p-2}\langle V(\nabla u), \nabla u\rangle d x \leq \mu \int_{\Omega}|\nabla u|^{p} d x=\mu \int_{\Omega} u f(u) d x .
$$

By $\operatorname{div} V(x)=\sum_{i=1}^{n} a_{i i}=n$, we obtain the following inequality:

$$
\begin{aligned}
& (p \mu-n) \int_{\Omega} u f(x, u) d x+p n \int_{\Omega} F(x, u) d x+p \int_{\Omega} F_{1}(x, u) d x \\
& \geq(p-1) \int_{\partial \Omega}\langle V(x), v(x)\rangle\left|\frac{\partial u}{\partial v}\right|^{p} d s .
\end{aligned}
$$

The proof is complete.

Based on the above Pohozaev-type inequality, we discuss the non-existence of a positive solution of the following boundary value problem:

$$
\begin{cases}-\Delta_{p} u=\lambda_{1}|u|^{r-2} u+\lambda_{2}|u|^{s-2} u, & \text { in } \Omega, \\ u=0, & \text { on } \partial \Omega\end{cases}
$$

where $1<r \leq s<+\infty$.

Theorem 2.3 Suppose that there exists a vector field $V(x)$ which satisfies the conditions of Theorem 2.2 and is transverse to $\partial \Omega$. Then (2.2) has no positive solution in the following cases respectively:

$\left(D_{1}\right) p_{1}^{*}>r, \lambda_{1}>0, \lambda_{2}>0, p_{1}^{*}>s$;

$\left(D_{2}\right) p_{1}^{*}>r, \lambda_{1}>0, \lambda_{2}<0, p_{1}^{*}<s$

$\left(D_{3}\right) p_{1}^{*}<r, \lambda_{1}<0, \lambda_{2}<0, p_{1}^{*}<s$,

where $p_{1}^{*}=\frac{n p}{n-p \mu}$.

Proof It is easy to see that

$$
f(x, u)=\lambda_{1}|u|^{r-2} u+\lambda_{2}|u|^{s-2} u, \quad F(x, u)=\int_{0}^{u} f(x, s) d s=\frac{\lambda_{1}}{r}|u|^{r}+\frac{\lambda_{2}}{s}|u|^{s},
$$

and then we have

$$
u f(x, u)=\lambda_{1}|u|^{r}+\lambda_{2}|u|^{s}, \quad \frac{\partial F(x, u)}{\partial x_{i}}=0, \quad F_{1}=0 .
$$


Suppose that $u$ is a positive solution of (2.2), according to Theorem 2.2, inequality (2.1) holds, then we have

$$
(p \mu-n) \int_{\Omega}\left(\lambda_{1}|u|^{r}+\lambda_{2}|u|^{s}\right) d x+p n \int_{\Omega} \frac{\lambda_{1}}{r}|u|^{r}+\frac{\lambda_{2}}{s}|u|^{s} d x \geq 0 .
$$

This yields

$$
\lambda_{1}\|u\|_{r}^{r}\left(\frac{r-p_{1}^{*}}{r}\right) \geq \lambda_{2}\|u\|_{s}^{s}\left(\frac{p_{1}^{*}-s}{s}\right) .
$$

By $\left(D_{1}\right)-\left(D_{3}\right)$, the left-hand side of (2.3) is negative, and the right-hand side is positive, which is a contradiction. Then the proof is complete.

Especially, we consider the problem in the case of $p=2$ :

$$
\begin{cases}-\Delta u=\lambda_{1}|u|^{r-2} u+\lambda_{2}|u|^{s-2} u, & \text { in } \Omega, \\ u=0, & \text { on } \partial \Omega .\end{cases}
$$

Corollary 2.4 Suppose that there exists a vector field $V(x)$ which satisfies the conditions of Theorem 2.2 and is transverse to $\partial \Omega$. Then (2.4) has no positive solution in the following cases respectively:

$\left(D_{1}^{\prime}\right) 2_{1}^{*}>r, \lambda_{1}>0, \lambda_{2}>0,2_{1}^{*}>s$

$\left(D_{2}^{\prime}\right) 2_{1}^{*}>r, \lambda_{1}>0, \lambda_{2}<0,2_{1}^{*}<s$;

$\left(D_{3}^{\prime}\right) 2_{1}^{*}<r, \lambda_{1}<0, \lambda_{2}<0,2_{1}^{*}<s$,

where $2_{1}^{*}=\frac{2 n}{n-2 \mu}$.

\section{The weighted quasi-linear elliptic boundary value problem}

This section is devoted to the weighted quasi-linear elliptic problem (1.2).

Theorem 3.1 (Pohozaev-type inequality) Let $V(x)$ be a linear vector field on $\mathbb{R}^{n}$ with the form

$$
V(x)=\left(\begin{array}{ccc}
a_{11} & \cdots & a_{1 n} \\
\vdots & \vdots & \vdots \\
a_{n 1} & \cdots & a_{n n}
\end{array}\right) x
$$

Suppose that $V(x)$ satisfies $\operatorname{div} V(x)=n$ and $\langle V(x), x\rangle>0$ for $\forall x \in \mathbb{R}^{n} \backslash\{0\}$. If $u \in W_{0}^{1, p}(\Omega) \cap$ $C^{1}(\bar{\Omega})$ is a solution of (1.2), then

$$
\begin{aligned}
& ((a+1) p \mu-n) \int_{\Omega} u f(x, u) d x+p n \int_{\Omega} F(x, u) d x+p \int_{\Omega} F_{1}(x, u) d x \\
& \geq(p-1) \int_{\partial \Omega}\langle V(x), v(x)\rangle\left|\frac{\partial u}{\partial v}\right|^{p} d s
\end{aligned}
$$

where $\mu=\sup _{|x| \neq 0} \frac{\langle V(x), x\rangle}{|x|^{2}}$. 
Proof We multiply equation (1.2) by $\langle V(x), \nabla u\rangle$, and then we integrate in $\Omega$. Similar to the proof of Theorem 2.2, by the divergence theorem and Lemma 2.1, we have

$$
\begin{aligned}
-\int_{\Omega} f(x, u)\langle V(x), \nabla u\rangle d x & =-\int_{\Omega} \sum_{i=1}^{n} f(x, u) V_{i}(x) \frac{\partial u}{\partial x_{i}} \\
& =\int_{\Omega} F(x, u) \operatorname{div} V(x) d x+\int_{\Omega} F_{1}(x, u) d x
\end{aligned}
$$

then

$$
\begin{aligned}
& =\sum_{j=1}^{n} \int_{\Omega} \frac{\partial}{\partial x_{j}}\left(|x|^{-a p}|\nabla u|^{p-2} \frac{\partial u}{\partial x_{j}}\right)\left(\sum_{i=1}^{n} V_{i}(x) \frac{\partial u}{\partial x_{i}}\right) d x \\
& =\sum_{j=1}^{n} \int_{\Omega} \frac{\partial}{\partial x_{j}}\left(|x|^{-a p}|\nabla u|^{p-2} \frac{\partial u}{\partial x_{j}} \sum_{i=1}^{n} V_{i}(x) \frac{\partial u}{\partial x_{i}}\right) \\
& -|x|^{-a p}|\nabla u|^{p-2} \frac{\partial u}{\partial x_{j}} \frac{\partial}{\partial x_{j}}\left(\sum_{i=1}^{n} V_{i}(x) \frac{\partial u}{\partial x_{i}}\right) d x \\
& =\int_{\partial \Omega}|x|^{-a p}|\nabla u|^{p}\langle V(x), v(x)\rangle d s \\
& -\sum_{j=1}^{n} \int_{\Omega}|x|^{-a p}|\nabla u|^{p-2} \frac{\partial u}{\partial x_{j}}\left(\sum_{i=1}^{n} a_{i j} \frac{\partial u}{\partial x_{i}}+\sum_{i=1}^{n} V_{i}(x) \frac{\partial^{2} u}{\partial x_{i} \partial x_{j}}\right) d x \\
& =\int_{\partial \Omega}\left\langle V(x),\left.\left.v(x)|| x\right|^{-a p}|| \nabla u\right|^{p} d s+\int_{\Omega} u \operatorname{div}\left(|x|^{-a p}|\nabla u|^{p-2} V(\nabla u)\right) d x\right. \\
& -\left.\sum_{j=1}^{n} \int_{\Omega}|x|^{-a p}|| \nabla u\right|^{p-2} \frac{\partial u}{\partial x_{j}}\left(\sum_{i=1}^{n} V_{i}(x) \frac{\partial^{2} u}{\partial x_{i} \partial x_{j}}\right) d x \\
& =\int_{\partial \Omega}\left\langle V(x),\left.v(x)|| x\right|^{-a p}|\nabla u|^{p} d s-\int_{\Omega}|x|^{-a p}|\nabla u|^{p-2}\langle V(\nabla u), \nabla u\rangle d x\right. \\
& -\frac{1}{p} \sum_{i=1}^{n} \int_{\Omega}|x|^{-a p} V_{i}(x) \frac{\partial}{\partial x_{i}}\left(|\nabla u|^{p}\right) d x \\
& =\int_{\partial \Omega}\left\langle V(x),\left.v(x)|| x\right|^{-a p}|\nabla u|^{p} d s-\int_{\Omega}|x|^{-a p}|\nabla u|^{p-2}\langle V(\nabla u), \nabla u\rangle d x\right. \\
& -\frac{1}{p} \int_{\partial \Omega}\left\langle V(x),\left.v(x)|| x\right|^{-a p}|\nabla u|^{p} d s+\frac{1}{p} \sum_{i=1}^{n} a_{i i} \int_{\Omega}|x|^{-a p}|\nabla u|^{p} d x\right. \\
& +\frac{1}{p} \sum_{i=1}^{n} \int_{\Omega}|\nabla u|^{p} V_{i} \frac{\partial}{\partial x_{i}}|x|^{-a p} d x \\
& =\int_{\partial \Omega}\left\langle V(x),\left.v(x)|| x\right|^{-a p}|\nabla u|^{p} d s-\int_{\Omega}|x|^{-a p}|\nabla u|^{p-2}\langle V(\nabla u), \nabla u\rangle d x\right. \\
& -\frac{1}{p} \int_{\partial \Omega}\langle V(x), v(x)\rangle|x|^{-a p}|\nabla u|^{p} d s+\frac{n}{p} \int_{\Omega}|x|^{-a p}|\nabla u|^{p} d x \\
& -a \int_{\Omega}|x|^{-a p-2}|\nabla u|^{p}\langle V(x), x\rangle d x \text {. }
\end{aligned}
$$


Since $0<\langle V(x), x\rangle \leq \mu|x|^{2}$, and $0<\langle V(\nabla u), \nabla u\rangle \leq \mu|\nabla u|^{2}$, we have

$$
\int_{\Omega}|\nabla u|^{p-2}\langle V(\nabla u), \nabla u\rangle d x \leq \mu \int_{\Omega}|\nabla u|^{p} d x=\mu \int_{\Omega} u f(u) d x .
$$

We obtain the following inequality:

$$
\begin{aligned}
& ((a+1) p \mu-n) \int_{\Omega} u f(x, u) d x+p n \int_{\Omega} F(x, u) d x+p \int_{\Omega} F_{1}(x, u) d x \\
& \geq(p-1) \int_{\partial \Omega}\langle V(x), v(x)\rangle\left|\frac{\partial u}{\partial v}\right|^{p} d s .
\end{aligned}
$$

The proof is complete.

Theorem 3.2 Suppose that there exists a vector field $V(x)$ which satisfies the conditions of Theorem 3.1 and is transverse to $\partial \Omega$. Then (1.2) has no positive solution if

$$
((a+1) p \mu-n) \int_{\Omega} u f(x, u) d x+p n \int_{\Omega} F(x, u) d x+p \int_{\Omega} F_{1}(x, u) d x<0 .
$$

Now we consider two special but important cases as follows.

1. A weighted quasi-linear problem

$$
\begin{cases}-\operatorname{div}\left(|x|^{-a p}|\nabla u|^{p-2} \nabla u\right)=\lambda_{1}|u|^{r-2} u+\lambda_{2}|u|^{s-2 u}, & \text { in } \Omega \\ u=0, & \text { on } \partial \Omega .\end{cases}
$$

By Theorem 3.2, problem (3.2) has no solution in the following cases.

Theorem 3.3 Suppose that the vector field $V(x)$ of Theorem 3.1 is transverse to $\partial \Omega$. Then (3.2) has no positive solution in the following cases respectively:

$\left(E_{1}\right) p_{2}^{*}>r, \lambda_{1}>0, \lambda_{2}>0, p_{2}^{*}>s$;

$\left(E_{2}\right) p_{2}^{*}>r, \lambda_{1}>0, \lambda_{2}<0, p_{2}^{*}<s$;

$\left(E_{3}\right) p_{2}^{*}<r, \lambda_{1}<0, \lambda_{2}<0, p_{2}^{*}<s$,

where $p_{2}^{*}=\frac{n p}{n-(a+1) p \mu}$.

2. A non-autonomous weighted quasi-linear problem:

$$
\begin{cases}-\operatorname{div}\left(|x|^{-a p}|\nabla u|^{p-2} \nabla u\right)=\lambda_{1}|x|^{-\alpha}|u|^{r-2} u+\lambda_{2}|x|^{-\beta}|u|^{s-2} u, & \text { in } \Omega \\ u=0, & \text { on } \partial \Omega .\end{cases}
$$

By Theorem 3.2, we have the next theorem of problem (3.3).

Theorem 3.4 Suppose that there exists a vector field $V(x)$ which satisfies the conditions of Theorem 2.3 and is transverse to $\partial \Omega$. If

$$
\left(\alpha_{0}-\frac{\alpha-n}{r}\right) \lambda_{1}<0, \quad \text { and } \quad\left(\frac{\beta-n}{s}-\alpha_{0}\right) \lambda_{2}>0
$$

where $\alpha_{0}=(a+1) \mu-\frac{n}{p}$, then (3.3) has no positive solution. 
Proof It is easy to see that

$$
\begin{aligned}
& f(x, u)=\lambda_{1}|x|^{-\alpha}|u|^{r-2} u+\lambda_{2}|x|^{-\beta}|u|^{s-2} u, \\
& F(x, u)=\int_{0}^{u} f(x, s) d s=\frac{\lambda_{1}}{r}|x|^{-\alpha}|u|^{r}+\frac{\lambda_{2}}{s}|x|^{-\beta}|u|^{s},
\end{aligned}
$$

and then we have

$$
u f(x, u)=\lambda_{1}|x|^{-\alpha}|u|^{r}+\lambda_{2}|x|^{-\beta}|u|^{s},
$$

and

$$
F_{1}(x, u)=\sum_{i=1}^{n} x_{i} \frac{\partial}{\partial x_{i}} F(x, u)=-\frac{\alpha \lambda_{1}}{r}|x|^{-\alpha}|u|^{r}-\frac{\beta \lambda_{2}}{s}|x|^{-\beta}|u|^{s} .
$$

Suppose that $u$ is a positive solution of (3.3), then inequality (3.1) holds, that is,

$$
\begin{aligned}
& ((a+1) p \mu-n) \int_{\Omega}\left(\lambda_{1}|x|^{-\alpha}|u|^{r}+\lambda_{2}|x|^{-\beta}|u|^{s}\right) d x+p n \int_{\Omega} F(x, u) d x+p \int_{\Omega} F_{1}(x, u) d x \\
& \geq(p-1) \int_{\partial \Omega}\langle V(x), v(x)\rangle\left|\frac{\partial u}{\partial v}\right|^{p} d s \geq 0 .
\end{aligned}
$$

This implies

$$
\begin{aligned}
& (p \mu-n) \int_{\Omega}\left(\lambda_{1}|x|^{-\alpha}|u|^{r}+\lambda_{2}|x|^{-\beta}|u|^{s}\right) d x+p n \int_{\Omega} \frac{\lambda_{1}}{r}|x|^{-\alpha}|u|^{r}+\frac{\lambda_{2}}{s}|x|^{-\beta}|u|^{s} d x \\
& \geq p \int_{\Omega} \frac{\alpha \lambda_{1}}{r}|x|^{-\alpha}|u|^{r}+\frac{\beta \lambda_{2}}{s}|x|^{-\beta}|u|^{s} d x .
\end{aligned}
$$

Hence, we get

$$
\lambda_{1}\left(\alpha_{0}-\frac{\alpha-n}{r}\right) \int_{\Omega}|x|^{-\alpha}|u|^{r} d x \geq \lambda_{2}\left(\frac{\beta-n}{s}-\alpha_{0}\right) \int_{\Omega}|x|^{-\beta}|u|^{s},
$$

which leads to a contradiction, because of (3.4).

Remark Let $\lambda_{1}=\lambda_{2}=-1, \alpha=b r, \beta=c s, r=\frac{n p}{n-p(a+1-b)}, s=\frac{n p}{n-p(a+1-c)}$, problem (3.3) is the equation that is discussed in [23].

\section{Acknowledgements}

We would like to express our great thanks to the referees for their valuable suggestions. This work was supported by the Fundamental Research Funds for the Central Universities (Grant No. 2015B37914).

\section{Competing interests}

The authors declare that they have no competing interests

Authors' contributions

Both authors contributed equally and significantly in writing this article. Both authors read and approved the final manuscript.

\section{Publisher's Note}

Springer Nature remains neutral with regard to jurisdictional claims in published maps and institutional affiliations.

Received: 1 February 2017 Accepted: 24 June 2017 Published online: 10 July 2017 


\section{References}

1. Pohozaev, S: Eigenfunctions of the equation $\Delta u+\lambda u=0$. Sov. Math. Dokl. 6, 1408-1411 (1965)

2. Dancer, EN, Zhang, K: Uniqueness of solutions for some elliptic equations and systems in nearly star-shaped domains. Nonlinear Anal. TMA 41, 745-761 (2000)

3. Druet, O, et al.: Stability of elliptic PDEs with respect to perturbations of the domain. J. Differ. Equ. 255, 3703-3718 (2013)

4. Fall, MM, Weth, T: Nonexistence results for a class of fractional elliptic boundary value problems. J. Funct. Anal. 263, 2205-2227 (2012)

5. Montenegro, M: On nontrivial solutions of critical polyharmonic elliptic systems. J. Differ. Equ. 247, 906-916 (2009)

6. Pucci, P, Serrin, J: A general variational identity. Indiana Univ. Math. J. 35, 681-703 (1986)

7. Reichel, W: Uniqueness results for semilinear polyharmonic boundary value problems on conformally contractible domains, I. J. Math. Anal. Appl. 287, 61-74 (2003)

8. Reichel, W: Uniqueness results for semilinear polyharmonic boundary value problems on conformally contractible domains, II. J. Math. Anal. Appl. 287, 75-89 (2003)

9. Wagner, A: Pohozaev identity from variational viewpoint. J. Math. Anal. Appl. 266, 149-159 (2002)

10. Bahri, A, Coron, L: On a nonlinear elliptic equation involving the critical Sobolev exponent: the effect of the topology of the domain. Commun. Pure Appl. Math. 41, 253-294 (1988)

11. Dancer, EN: A note on an equation with critical exponent. Bull. Lond. Math. Soc. 20,600-602 (1988)

12. Ding, W: Positive solutions of $\Delta u+u^{(n+2) /(n-2)}=0$ on contractible domains. J. Partial Differ. Equ. 2, 83-88 (1989)

13. Guedda, M, Veron, L: Quasilinear elliptic equations involving critical exponents. Nonlinear Anal. 1318, 879-902 (1989)

14. Isaia, F: Superposition operators between Sobolev spaces and a non-existence result of higher-order regular solutions for the p-Laplacian. Nonlinear Anal. 117(10), 87-98 (2015)

15. Takáč, $P, I$ Il'yasov, YS: Optimal $W_{\text {loc }}^{2,2}$-regularity, Pohozhaev's identity, and nonexistence of weak solutions to some quasilinear elliptic equations. J. Differ. Equ. 252, 2792-2822 (2012)

16. Pucci, P, Servadei, R: On weak solutions for $p$-Laplacian equations with weights. Atti Accad. Naz. Lincei, Rend. Lincei, Mat. Appl. 118, 257-267 (2007)

17. Pucci, P, Servadei, R: Regularity of weak solutions of homogeneous and inhomogeneous quasi-linear elliptic equations. Indiana Univ. Math. J. 57(7), 3329-3363 (2008)

18. Bartsch, T, Peng, S, Zhang, Z: Existence and non-existence of solutions to elliptic equations related to the Caffarelli-Kohn-Nirenberg inequalities. Calc. Var. Partial Differ. Equ. 30, 113-136 (2007)

19. Ambrosetti, A, Brézis, H, Cerami, G: Combined effects of concave and convex nonlinearities in some elliptic problems. J. Funct. Anal. 122, 519-543 (1994)

20. Lair, AV, Wood, AW: Large solutions of sublinear elliptic equations. Nonlinear Anal. 39, 745-753 (2000)

21. Lair, AV: Large solutions of semilinear elliptic equations under the Keller-Osserman condition. J. Math. Anal. Appl. 328 1247-1254 (2007)

22. Santos, CA: Nonexistence and existence of entire solutions for a quasilinear problem with singular and super-linear terms. Nonlinear Anal. 72, 3813-3819 (2010)

23. Xuan, BJ, Wang, J: Existence of a nontrivial weak solution to quasilinear elliptic equations with singular weights and multiple critical exponents. Nonlinear Anal. 72, 3649-3658 (2010)

\section{Submit your manuscript to a SpringerOpen ${ }^{\circ}$ journal and benefit from:}

- Convenient online submission

- Rigorous peer review

- Open access: articles freely available online

- High visibility within the field

- Retaining the copyright to your article

Submit your next manuscript at $\gg$ springeropen.com 